\title{
SUBGALEAL MIGRATION OF THE DISTAL CATHETER OF A VENTRICULOPERITONEAL SHUNT
}

\begin{abstract}
Fatih Serhat Erol, Bekir Akgun
Firat University, Faculty of Medicine, Elazig, Turkey: Department of Neurosurgery

Summary: Proximal migration of the distal end of a ventriculoperitoneal shunt has been observed much more rarely than other numerous shunt-related complications. Subgaleal migration of the peritoneal end is one of the samples. In the preset report we have discussed a case of subgaleal migration of the peritoneal end detected as a result of the examinations performed for shunt dysfunction. There was ventricular dilatation on CT scan of the brain. X-ray examinations confirmed proper ventricular catheter and shunt valve placement but a complete migration of distal (peritoneal) catheter into the subgaleal space. Then the patient's shunt was revised. When our case and the literature were examined, we observed that this complication was frequently encountered during the first postoperative months, in the pediatric ages and in patients with advanced hydrocephalus. Besides, we have detected that the peritoneal catheters had tendency to migration into the subgaleal tissues similar to pre-insertion forms of the preoperatively original packages.
\end{abstract}

Key words: Hydrocephalus; Ventriculoperitoneal shunt; Subgaleal migration

\section{Introduction}

Migration of the distal end of the ventriculoperitoneal (VP) shunt to different regions such as the peritoneal cavity, gall bladder, scrotum and its fracture and disconnection have been frequently reported in the literature $(4,5,7)$. The proximal migrations of the peritoneal end without any disconnection or fracture, such as the scalp migration, gathering subgaleally and migration into the ventricles or subdural space are rarer entities $(1,2,3,4,6)$.

We have presented our management of development of shunt dysfunction due to the subgaleal migration of the peritoneal end in a patient and discussed the possible mechanisms, risk factors, detection age and period of the complication with accompaniment with the literature in this present study.

\section{Case report}

A 5-month-old girl was admitted to our clinic with complaints of vomiting, agitation and inability to eat. She has an episode of excision of the myelomeningocele sac and receiving ventriculoperitoneal shunt surgery for hydrocephalus at 2 months of age. "Setting sun sign" of the eyes and dilatation of the scalp veins were detected in the neurological examination. Anterior fontanel was observed to be convex and tight. Head circumference was measured $52 \mathrm{~cm}$ (>97 P) (Head circumference was $45 \mathrm{~cm}$ at discharge 3 months ago). There was ventricular dilatation on cranial CT. X-ray examinations confirmed that distal shunt catheter was not observed in the chest and abdominal graphics (Fig. 1). Ventricular catheter and shunt valve were detected to be in the correct placement; however, the migration of the distal (peritoneal) catheter completely towards subgaleal space was observed (Fig. 2). Subsequently, the patient's shunt was revised. No problems were noted during the regular follow-up.

\section{Discussion}

Application of VP shunt as a treatment for symptomatic hydrocephalus is the most frequently preferred treatment method $(1,4)$. There are various types of shunt complications. An important point is that the causes of shunt complications are frequently multifactor (4). Consequently, we are in the opinion that reporting especially the rarely seen complications and assessment of the possible causes in the light of the literature are important for reducing shunt-related complications or for the early and correct diagnosis and treatment of the developing shunt complications.

Ventricular or peritoneal catheter disconnections, abdominal migrations are the vp shunt complications which are frequently reported $(4,5,7)$. Proximal migration of the peritoneal end without any disconnection or fracture is much more rarely observed $(1,2,3,4,6)$. 


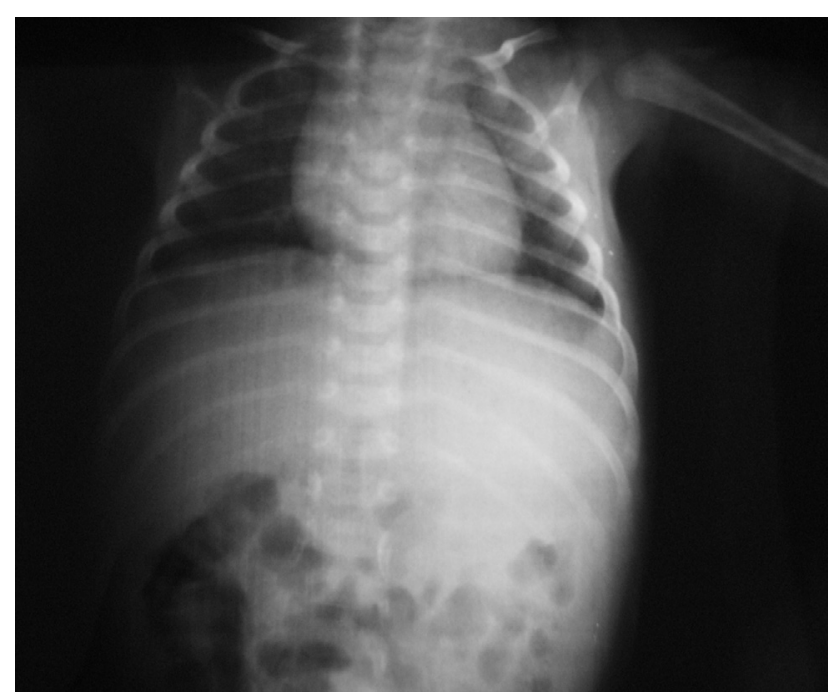

Fig. 1: No distal shunt catheter is observed as a result of the chest and abdominal X-ray examinations.

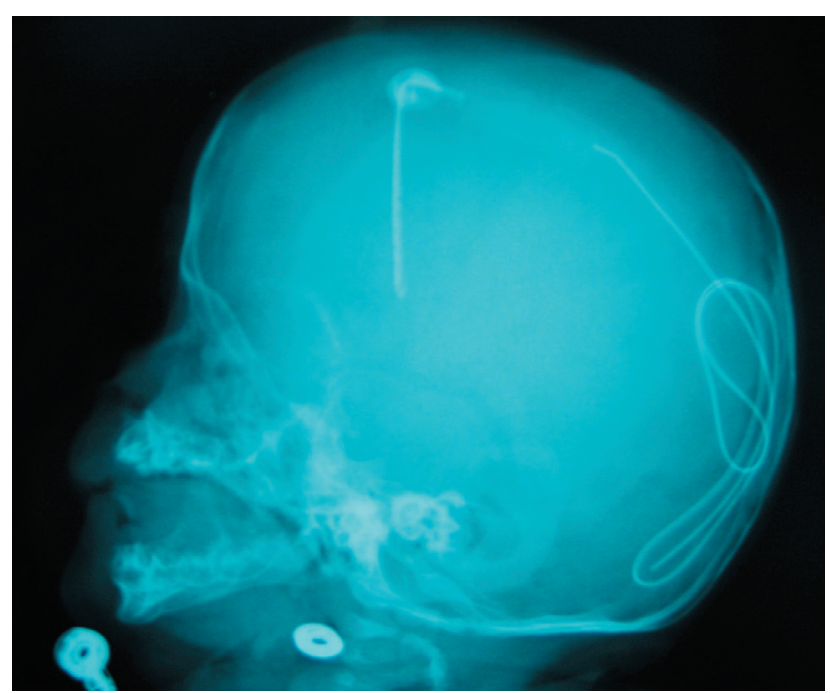

Fig. 2: Ventricular catheter and shunt valve are observed to be correctly placed but a complete migration of the distal (peritoneal) catheter to the subgaleal space is observed in the head graph.

Various hypotheses on the proximal migration of the peritoneal end have been presented. An important opinion is that the flexion and extension motions of the patient's head may enable pulling the peritoneal catheter upward $(1,4,6)$. Inadequate fixations and the excessive soft-tissue dissection during surgery are presented also among the possible causes (4).

An important point attracting attention is that this complication is frequently observed in pediatric patients, who are especially in early childhood (6). Shunt fractures, disconnections and abdominal migrations are observed both in early childhood, and primary school age, which are the rapidly growing period in height and weight; however, when we reviewed the cases published in the literature we determined that the proximal migration frequently began in early childhood. These facts make us consider that these complications have different mechanisms. The stretching of the distal catheter between the valve and the abdomen during the rapidly growing period have been thought to be one of the basic causes of fracture, disconnection and abdominal migration $(5,8)$. It has been guessed that the supine position during breast-feeding and the presence of loose subcutaneous tissue that may accompany this in the child may facilitate the complication in proximal migration $(1,6)$.

Proximal migration is more frequently observed in the ventriculoperitoneal shunts than those placed into the pleural or atrial cavities. This is why various writers suspected from the high mobility of the peritoneal catheter inside the abdomen and associated anatomical characteristics of the abdominal cavity (6).

The presence of positive intra-abdominal pressure against negative intraventricular pressure is one of the believed causes. Conditions, especially in which a peritoneal absorption secondary to an inflammatory reaction may be spoiled or the formation of peritoneal cyst which may cause high pressure pushing the distal catheter may be observed in patients with VP shunt (6). Actually, CSF pressure of the patients with extremely open fontanel due to advanced hydrocephalus and with open fontanel despite having no intra-abdominal pathology is close to the atmospheric pressure. The intra-abdominal pressure is positive with respect to the atmosphere and this may have a pressure-regulating effect. The peritoneal catheters of such patients are naturally exposed to high abdominal pressure (6). This information suggests us a possible correlation between the ventricular dilatation amount, intensity of hydrocephalus or overmeasurement of the patient's head circumference and the prevalence risk of this complication. In fact, we believe that this is one of the causes contributing the development of the complication because the head circumference of our subject was greater than the $97^{\text {th }}$ percentile since the first shunt surgery and the amount of ventricular dilatation on CT was too much and the cerebral parenchymal tissue was too thin.

In a study Dominguez et al. (1) reported this cause as depending on the packaging of the shunt system in a tightly coiled fashion and predisposition plastic pipe-like system to be collected in coils after the insertion to the patient. Pre-insertion, the shunt was in a tightly coiled form in the package and 2 months after the patient was supplied with the shunt it was lying in a similar tightly coiled fashion in the subgaleal tissue. The pre-insertion packed form of the shunt used for our patient was also in the form of a coil but it was not coiled tightly. Besides, the catheter inserted into the subgaleal tissue demonstrated loose coiling which is suggested to be caused by the tightly coiled shunts in the packaging but it also suggests that as a result of this complication shunts have tendency for migration similar to pre- 
insertion forms though not packaged in tightly coiled fashion. When examined in the light of the literature, the abdominal migrations and shunt disconnections are mostly late period shunt complications, but the proximal migrations are early post operative period shunt complications occurring until the $3^{\text {rd }}$ month $(2,3)$. This is a good indicator showing that the shunt tube is predisposed to return to the appearance in the memory, that is, the packaged form in the early period.

\section{Conclusion}

In conclusion, though seen infrequently, after the VP shunt applications, the peritoneal catheter may move into the subgaleal tissue following the proximal migration of the peritoneal catheter. Due this shunt dysfunction and revision requirement may be observed in the patient. When our case and the literature are evaluated, in patients with shunt dysfunction, especially in postoperative first months, pediatric ages and in patients with advanced hydrocephalus, such a complication should be remembered. For early and accurate diagnosis and treatment planning x-rays, rapid, noninvasive and cheap methods, should be taken. Another attractive point is that the peritoneal catheter is predisposed to a migration similar in appearance to that of the pre-insertion to the subgaleal tissue, in other words, similar in appearance to the one in its memory.

\section{References}

1. Dominguez CJ, Tyagi A, Hall G, Timothy J, Chumas PD. Sub-galeal coiling of the proximal and distal components of a ventriculo-peritoneal shunt. An unusual complication and proposed mechanism. Childs Nerv Syst 2000;16:493-5.

2. Eljamel MS, Sharif S, Pidgeon CN. Total intraventricular migration of unisystem ventriculo-peritoneal shunt. Acta Neurochirur (Wien) 1995;136:217-18.

3. Gupta PK, Dev EJ, Lad SD. Total migration of a ventriculo-peritoneal shunt into the ventricles. Br J Neurosurg 1999;13:73-4.

4. Hinai QSA, Pawar SJ, Sharma RR, Devadas RV. Subgaleal migration of a ventriculoperitoneal shunt. J Clin Neurosci 2006;13:666-9.

5. Kaplan M, Ozel SK, Donmez O, Kazez A. Treatment approaches for abdominal migration of peritoneal catheter of ventriculoperitoneal shunt. Turk Neurosurg 2007;17(2):158-62

6. Nadkarni TD, Menon RK, Dange NN, Desai KI, Goel A. Cranial migration of complete ventriculo-peritoneal shunt assembly. J Clin Neurosci 2007;14:92-4.

7. Ozveren MF, Kazez A, Cetin H, Ziyal IM. Migration of the abdominal catheter of a ventriculoperitoneal shunt in to the scrotum: case report. Neurol Med Chir (Tokyo) 1999;39:313-15

8. Tsurushima H, Harakuni T, Saito A, Hyodo A, Yoshii Y. Shunt tube problems due to placement of valves on the chest wall: three case reports. Neurol Med Chir (Tokyo) 2000;40:342-4.

\section{Corresponding author:}

Bekir Akgun, M.D, Firat University, Tip Fakultesi, Firat Tip Merkezi, Beyin Cerrahi Klinigi, 23119, Elazig, Turkey; e-mail: bekirakgun@yahoo.com 